\title{
Breit Equation with Form Factors in the Hydrogen Atom
}

\author{
F. García Daza, N. G. Kelkar and M. Nowakowski \\ Departamento de Fisica, Universidad de los Andes, \\ Cra.1E No.18A-10, Bogota, Colombia
}

\begin{abstract}
The Breit equation with two electromagnetic form-factors is studied to obtain a potential with finite size corrections. This potential with proton structure effects includes apart from the standard Coulomb term, the Darwin term, retarded potentials, spin-spin and spin-orbit interactions corresponding to the fine and hyperfine structures in hydrogen atom. Analytical expressions for the hyperfine potential with form factors and the subsequent energy levels including the proton structure corrections are given using the dipole form of the form factors. Numerical results are presented for the finite size corrections in the $1 \mathrm{~S}$ and $2 \mathrm{~S}$ hyperfine splittings in the hydrogen atom, the Sternheim observable $D_{21}$ and the $2 \mathrm{~S}$ and $2 \mathrm{P}$ hyperfine splittings in muonic hydrogen. Finally, a comparison with some other existing methods in literature is presented.

PACS numbers: 03.65.-w, 32.10.Fn, 13.40.Gp
\end{abstract}




\section{INTRODUCTION}

The Breit equation [1, 2] is a paradigm example of how one derives coordinate potentials from Quantum Field Theory: an elastic scattering amplitude, expanded in powers of $1 / c^{2}$ and depending on the three momentum transfer $\mathbf{q}$, gets Fourier transformed into the coordinate space. The result is the potential $V(r)$. Famous examples of this general principle include, among others, (i) the Casimir-Polder forces between neutral atoms as van der Waals forces at large distances from a two photon exchange amplitude [3], (ii) the Uehling-Serber potential from one-loop vacuum polarization diagram [4], (iii) the Feinberg-Sucher two neu-

trino exchange force [5], (iv) the microscopic potential of Nuclear Physics based on $\sigma, \rho$ and $\omega$ exchanges [6] and (v) quantum corrections to the Newtonian potential [7]. Other potentials are derived from pseudoscalar (Goldstone bosons) exchanges [8], scalar Higgs exchanges (Higgsonium) [9] and even from Finite Temperature Quantum Field Theory which gives temperature dependent potentials [10]. The span of the applications of these potentials, derived via the Fourier transform of an elastic amplitude, ranges from atomic and molecular physics up to nuclear-particle physics and even gravitation and cosmology [11].

The Breit equation follows the very same principle for elastic $e^{-} \mu^{+}, e^{+} e^{-}$(positronium), $e^{-} p$ (hydrogen) and $\mu^{-} p$ (muonic hydrogen) amplitudes. The outcome is a potential which goes beyond the Coulomb potential and includes the correct expressions for fine structure, hyperfine structure and the Darwin terms. To appreciate this fact let us mention that the fine and hyperfine structure Hamiltonians in non-relativistic Quantum Mechanics are derived by using semi-classical arguments [12] whereas the Breit equation does so automatically in a systematic way without reference to semi-classical arguments. This offers more insight into semi-relativistic two body Hamiltonians. For instance, the standard Breit equation for two spin-1/2 particles can be extended to a spin-1/2 spin-0 system [13] necessary for exotic atoms. Higher order terms in the $1 / c^{2}$ expansion can be taken into account and as we will show later, the finite size corrections can be taken into account in a straightforward way. Indeed, the finite size corrections to the potential have to do with the way the photon interacts with an extended particle, i.e., with a mild modification of the vertex for point-like particles (which is to say that the modified vertex will now include the form-factors). Hence, the modification of the vertices in the elastic amplitudes leads after a Fourier transform to a potential which takes into account the finite size corrections. This is a straightforward 
generalization of the standard Breit equation where the finite size corrections are included in a natural way using a one-photon exchange diagram. The finite size corrections to the potential can then be applied to calculate the finite size corrections to the energy levels of the electronic or muonic hydrogen atom. The necessity to do so is the ever increasing accuracy of the theoretical QED corrections to the energy levels and the accurate experimental results.

The phenomenology of the hydrogen atom cannot be disentangled from the historical development of Quantum Mechanics (QM). Indeed, any progress in QM, going from nonrelativistic to relativistic QM and finally to relativistic Quantum Field Theory, was accompanied by new corrections to the energy levels of the hydrogen atom and its cousin the muonic hydrogen $\left(\mu^{-} p\right.$, where a negative muon replaces the electron in hydrogen). The hydrogen atom thus became a precision tool for testing predictions of Quantum Electrodynamics (QED) and more generally of electroweak theory, including symmetry considerations [14]. The experimental techniques have reached an extraordinary precision [15] which sometimes surpasses the theoretically calculated values expanded in the fine structure constant $\alpha$. For example, one of the most precisely measured quantities in physics is the hyperfine structure (hfs) of the ground state of hydrogen atom [16], which is known to one part in $10^{13}$. In spite of this progress, there still remains one component, namely, the structure of the proton in the hydrogen atom which introduces uncertainties in the comparison of theories with experiment. Here, we demonstrate that the precision values calculated using QED get blurred by the nuclear finite size corrections (FSC). We focus in particular on these corrections in the hfs of hydrogen and $\mu^{-} p$. We use the theoretical framework of the Breit equation which in the case of the electromagnetic (EM) form factors taken at zero momentum transfer gives the standard hyperfine Hamiltonian. The frequencies of the hyperfine intervals for the $(1 S)$ and $(2 S)$ levels in hydrogen are first evaluated using the Breit equation with and without the EM form factors and compared with the precise experimental values. The proton structure correction to the Sternheim hyperfine interval [17] $D_{21}=8 E_{h f s}(2 S)-E_{h f s}(1 S)$ is evaluated and found to be much smaller than that obtained using the Zemach method [18]. Finally, the FSC for the hfs in $\mu^{-} p$ which was used as an input for calculating the proton radius $\left(r_{p}\right)$ in a recent accurate claim of the measurement of $r_{p}$ [19] are also evaluated. The above calculations show that the accurate measurements are not just limited due to the structure corrections and uncertainties in the proton form factors but also depend on the approach for the finite size corrections. 
In the next section, we introduce the framework of the Breit equation and derive the potential with finite size corrections due to the structure of the proton. After having presented the full Breit potential with form factors, in section III we focus on the part of the potential which corresponds to the hyperfine structure in hydrogen atom. The expressions for the corrections to the energy levels are evaluated using time independent perturbation theory. In section IV, we present the numerical results for hyperfine splitting in electronic $\left(e^{-} p\right)$ and muonic $\left(\mu^{-} p\right)$ hydrogen and compare them with available data as well as results in literature obtained using other methods to incorporate the proton structure effects. The relevance of the results of the present work is summarized in the last section.

\section{THE BREIT POTENTIAL}

To start with, we briefly introduce the framework of the Breit equation. It involves an expansion of the elastic scattering amplitude, say, $e p \rightarrow e p$ in powers of $1 / c^{2}$, thus having the advantage of systematically taking into account the relativistic corrections. Starting with the one photon exchange Feynman diagram for a system of two point-like spin 1/2 particles (like $e^{-} \mu^{+}$for example), the full Hamiltonian for the $e^{-} p$ system considering pointlike protons can be written as,

$$
\hat{H}=\hat{H}_{e}^{(0)}+\hat{H}_{p}^{(0)}+\hat{U}\left(\hat{\mathbf{p}}_{e}, \hat{\mathbf{p}}_{p}, \mathbf{r}\right)
$$

where it is convenient to split the potential into several parts (to be discussed below):

$$
\hat{U}\left(\hat{\mathbf{p}}_{e}, \hat{\mathbf{p}}_{p}, \mathbf{r}\right)=\sum_{i=1}^{11} \hat{V}_{i}\left(\hat{\mathbf{p}}_{e}, \hat{\mathbf{p}}_{p}, \mathbf{r}\right) .
$$

The free Hamiltonian is expanded up to the same order in $1 / c$ as the potential. In our case we have for example,

$$
\hat{H}_{e, p}^{0}=\frac{\hat{\mathbf{p}}_{e, p}^{2}}{2 m_{e, p}}-\frac{\hat{\mathbf{p}}_{e, p}^{4}}{8 m_{e, p}^{3} c^{2}} .
$$

The potential $\hat{U}\left(\mathbf{p}_{e}, \mathbf{p}_{p}, \mathbf{q}\right)$ in momentum space is obtained by writing the elastic amplitude $M_{f i}$ in terms of two-component spinors $w_{e, p}$, i.e.,

$$
M_{f i}=-2 m_{e} \cdot 2 m_{p}\left(w_{e}^{\prime *} w_{p}^{\prime *}\right) \hat{U}\left(\mathbf{p}_{e}, \mathbf{p}_{p}, \mathbf{q}\right)\left(w_{e} w_{p}\right) .
$$

The Fourier transform of $\hat{U}\left(\mathbf{p}_{e}, \mathbf{p}_{p}, \mathbf{q}\right)$ is the potential $\hat{U}\left(\hat{\mathbf{p}}_{e}, \hat{\mathbf{p}}_{p}, \mathbf{r}\right)$ :

$$
\hat{U}\left(\hat{\mathbf{p}}_{e}, \hat{\mathbf{p}}_{p}, \mathbf{r}\right)=\int e^{i \mathbf{q} \cdot \mathbf{r}} \hat{U}\left(\mathbf{p}_{e}, \mathbf{p}_{p}, \mathbf{q}\right) \frac{d^{3} q}{(2 \pi)^{3}},
$$


where in the center of mass system we can identify $\hat{\mathbf{p}}_{e}=-\hat{\mathbf{p}}_{p}=-i \nabla$. The standard result for the potential in momentum space is [25],

$$
\begin{aligned}
& \hat{U}\left(\mathbf{p}_{e}, \mathbf{p}_{p}, \mathbf{q}\right)=4 \pi e^{2}\left(-\frac{1}{\mathbf{q}^{2}}+\frac{1}{8 m_{e}^{2} c^{2}}+\frac{1}{8 m_{p}^{2} c^{2}}+\frac{i \boldsymbol{\sigma}_{p} \cdot\left(\mathbf{q} \times \mathbf{p}_{p}\right)}{4 m_{p}^{2} c^{2} \mathbf{q}^{2}}-\frac{i \boldsymbol{\sigma}_{e} \cdot\left(\mathbf{q} \times \mathbf{p}_{e}\right)}{4 m_{e}^{2} c^{2} \mathbf{q}^{2}}\right. \\
& +\frac{\mathbf{p}_{e} \cdot \mathbf{p}_{p}}{m_{e} m_{p} c^{2} \mathbf{q}^{2}}-\frac{\left(\mathbf{p}_{e} \cdot \mathbf{q}\right)\left(\mathbf{p}_{p} \cdot \mathbf{q}\right)}{m_{e} m_{p} c^{2} \mathbf{q}^{4}}-\frac{i \boldsymbol{\sigma}_{p} \cdot\left(\mathbf{q} \times \mathbf{p}_{e}\right)}{2 m_{e} m_{p} c^{2} \mathbf{q}^{2}}+\frac{i \boldsymbol{\sigma}_{e} \cdot\left(\mathbf{q} \times \mathbf{p}_{p}\right)}{2 m_{e} m_{p} c^{2} \mathbf{q}^{2}}+\frac{\boldsymbol{\sigma}_{e} \cdot \boldsymbol{\sigma}_{p}}{4 m_{e} m_{p} c^{2}} \\
& \left.-\frac{\left(\boldsymbol{\sigma}_{e} \cdot \mathbf{q}\right)\left(\boldsymbol{\sigma}_{p} \cdot \mathbf{q}\right)}{4 m_{e} m_{p} c^{2} \mathbf{q}^{2}}\right),
\end{aligned}
$$

and the individual terms of the potential in coordinate space come out to be,

$$
\begin{aligned}
& \hat{V}_{1}=-\frac{e^{2}}{r} \\
& \hat{V}_{2}=\frac{\pi e^{2}}{2 m_{e}^{2} c^{2}} \delta(\mathbf{r}) \\
& \hat{V}_{3}=\frac{\pi e^{2}}{2 m_{p}^{2} c^{2}} \delta(\mathbf{r}) \\
& \hat{V}_{4}=-\frac{e^{2}}{4 m_{p}^{2} c^{2}} \frac{\left(\mathbf{r} \times \hat{\mathbf{p}}_{p}\right)}{r^{3}} \cdot \boldsymbol{\sigma}_{p}, \\
& \hat{V}_{5}=\frac{e^{2}}{4 m_{e}^{2} c^{2}} \frac{\left(\mathbf{r} \times \hat{\mathbf{p}}_{e}\right)}{r^{3}} \cdot \boldsymbol{\sigma}_{e}, \\
& \hat{V}_{6}=\frac{e^{2}}{m_{e} m_{p} c^{2}} \frac{\hat{\mathbf{p}}_{e} \cdot \hat{\mathbf{p}}_{p}}{r}, \\
& \hat{V}_{7}=-\frac{e^{2}}{2 m_{e} m_{p} c^{2} r}\left(\hat{\mathbf{p}}_{e} \cdot \hat{\mathbf{p}}_{p}-\frac{\mathbf{r} \cdot\left(\mathbf{r} \cdot \hat{\mathbf{p}}_{e}\right) \hat{\mathbf{p}}_{p}}{r^{2}}\right) \\
& \hat{V}_{8}=\frac{e^{2}}{2 m_{e} m_{p} c^{2}} \frac{\left(\mathbf{r} \times \hat{\mathbf{p}}_{e}\right)}{r^{3}} \cdot \boldsymbol{\sigma}_{p}, \\
& \hat{V}_{9}=-\frac{e^{2}}{2 m_{e} m_{p} c^{2}} \frac{\left(\mathbf{r} \times \hat{\mathbf{p}}_{p}\right)}{r^{3}} \cdot \boldsymbol{\sigma}_{e}, \\
& \hat{V}_{10}=\frac{\pi e^{2}}{m_{e} m_{p} c^{2}}\left(\boldsymbol{\sigma}_{e} \cdot \boldsymbol{\sigma}_{p}\right) \delta(\mathbf{r}), \\
& \hat{V}_{11}=-\frac{e^{2}}{4 m_{e} m_{p} c^{2}}\left(\frac{\boldsymbol{\sigma}_{e} \cdot \boldsymbol{\sigma}_{p}}{r^{3}}-3 \frac{\left(\boldsymbol{\sigma}_{e} \cdot \mathbf{r}\right)\left(\boldsymbol{\sigma}_{p} \cdot \mathbf{r}\right)}{r^{3}}+\frac{4 \pi}{3} \boldsymbol{\sigma}_{e} \cdot \boldsymbol{\sigma}_{p} \delta(\mathbf{r})\right) .
\end{aligned}
$$

Here $e^{2}=\alpha$. The meaning of the terms is as follows:

- $\hat{V}_{1}$ is obviously the Coulomb potential

- $\hat{V}_{2}$ and $\hat{V}_{3}$ are the Darwin terms which are also present in the Dirac equation [20] 
- $\hat{V}_{6}, \hat{V}_{7}$ are called retarded potentials

- $\hat{V}_{5}$ and $\hat{V}_{9}$ are the spin-orbit interaction terms which give rise to the fine structure; $\hat{V}_{4}$ and $\hat{V}_{8}$ are also a spin-orbit terms, however, traditionally added to the hyperfine part [21]

- $\hat{V}_{10}, \hat{V}_{11}$ are the standard terms of the hyperfine Hamiltonian.

It is clear that the hyperfine part given above is valid only for point-like particles. For the electron-proton system we miss the correct magnetic moment of the proton. Indeed, for the hydrogen atom, the standard hyperfine potential as in text books [12] reads in natural units, $\hbar=c=1$,

$$
\hat{V}_{\mathrm{hfs}}=\frac{\alpha}{m_{e} m_{p} c^{2}} \frac{g_{p}}{2}\left[\frac{3\left(\mathbf{S}_{e} \cdot \hat{\mathbf{r}}\right)\left(\mathbf{S}_{e} \cdot \hat{\mathbf{r}}\right)-\mathbf{S}_{e} \cdot \mathbf{S}_{p}}{r^{3}}+\frac{8 \pi}{3} \mathbf{S}_{e} \cdot \mathbf{S}_{p} \delta^{3}(\mathbf{r})\right]
$$

with $g_{p}=5.58$. Replacing

$$
\mathbf{S}_{e, p}=\boldsymbol{\sigma}_{e, p} / 2
$$

and comparing with Eq. (17), one can see that $\hat{V}_{11}$ in (17) gives the hyperfine potential, however, without the factor $g_{p} / 2$. The missing factor in the Breit Hamiltonian of Eq. (17) is part of the second form-factor of the proton, namely,

$$
\left[1+\kappa_{p}\right]=\left[1+F_{2}^{p}(0)\right]=g_{p} / 2 .
$$

The purpose of this small exercise is to bring to the reader's attention that for point-like particles, $F_{2}(0)$ is very small (and hence $g_{p} \approx 1$ in the Breit potential in (17)), as this quantity arises from one and more loops (anomalous magnetic moment). The hyperfine potential in (18) already includes part of the finite size corrections (in the form of $g_{p}=2\left[1+F_{2}^{p}(0)\right]$ ). To remedy the situation for the $e^{-} p$ system in the Breit equation we enlarge it by allowing the electromagnetic form-factors in the vertex for the proton (and for the sake of generality also for the electron) as follows:

$$
\bar{u}\left(p^{\prime}\right) \Gamma^{\mu}\left(p^{\prime}, p\right) u(p)=\bar{u}\left(p^{\prime}\right)\left(\gamma^{\mu} F_{1}\left(q^{2}\right)+\frac{i}{2 m_{p}} F_{2}\left(q^{2}\right) \sigma^{\mu \nu} q_{\nu}\right) u(p) .
$$

Doing so, we obtain the following potential in momentum space [13]

$$
\hat{U}\left(\mathbf{p}_{e}, \mathbf{p}_{p}, \mathbf{q}\right)=4 \pi e^{2}\left[F _ { 1 } ^ { e } F _ { 1 } ^ { p } \left(-\frac{1}{\mathbf{q}^{2}}+\frac{1}{8 m_{e}^{2} c^{2}}+\frac{1}{8 m_{p}^{2} c^{2}}+\frac{i \boldsymbol{\sigma}_{p} \cdot\left(\mathbf{q} \times \mathbf{p}_{p}\right)}{4 m_{p}^{2} c^{2} \mathbf{q}^{2}}-\frac{i \boldsymbol{\sigma}_{e} \cdot\left(\mathbf{q} \times \mathbf{p}_{e}\right)}{4 m_{e}^{2} c^{2} \mathbf{q}^{2}}\right.\right.
$$




$$
\begin{aligned}
& +\frac{\mathbf{p}_{e} \cdot \mathbf{p}_{p}}{m_{e} m_{p} c^{2} \mathbf{q}^{2}}-\frac{\left(\mathbf{p}_{e} \cdot \mathbf{q}\right)\left(\mathbf{p}_{p} \cdot \mathbf{q}\right)}{m_{e} m_{p} c^{2} \mathbf{q}^{4}}-\frac{i \boldsymbol{\sigma}_{p} \cdot\left(\mathbf{q} \times \mathbf{p}_{e}\right)}{2 m_{e} m_{p} c^{2} \mathbf{q}^{2}}+\frac{i \boldsymbol{\sigma}_{e} \cdot\left(\mathbf{q} \times \mathbf{p}_{p}\right)}{2 m_{e} m_{p} c^{2} \mathbf{q}^{2}}+\frac{\boldsymbol{\sigma}_{e} \cdot \boldsymbol{\sigma}_{p}}{4 m_{e} m_{p} c^{2}} \\
& \left.-\frac{\left(\boldsymbol{\sigma}_{e} \cdot \mathbf{q}\right)\left(\boldsymbol{\sigma}_{p} \cdot \mathbf{q}\right)}{4 m_{e} m_{p} c^{2} \mathbf{q}^{2}}\right)+F_{1}^{e} F_{2}^{p}\left(\frac{1}{4 m_{p}^{2} c^{2}}+\frac{i \boldsymbol{\sigma}_{p} \cdot\left(\mathbf{q} \times \mathbf{p}_{p}\right)}{2 m_{p}^{2} c^{2} \mathbf{q}^{2}}-\frac{i \boldsymbol{\sigma}_{p} \cdot\left(\mathbf{q} \times \mathbf{p}_{e}\right)}{2 m_{e} m_{p} c^{2} \mathbf{q}^{2}}-\frac{\left(\boldsymbol{\sigma}_{e} \cdot \mathbf{q}\right)\left(\boldsymbol{\sigma}_{p} \cdot \mathbf{q}\right)}{4 m_{e} m_{p} c^{2} \mathbf{q}^{2}}\right. \\
& \left.+\frac{\boldsymbol{\sigma}_{e} \cdot \boldsymbol{\sigma}_{p}}{4 m_{e} m_{p} c^{2}}\right)+F_{2}^{e} F_{1}^{p}\left(\frac{1}{4 m_{e}^{2} c^{2}}-\frac{i \boldsymbol{\sigma}_{e} \cdot\left(\mathbf{q} \times \mathbf{p}_{e}\right)}{2 m_{e}^{2} c^{2} \mathbf{q}^{2}}+\frac{i \boldsymbol{\sigma}_{e} \cdot\left(\mathbf{q} \times \mathbf{p}_{p}\right)}{2 m_{e} m_{p} c^{2} \mathbf{q}^{2}}-\frac{\left(\boldsymbol{\sigma}_{e} \cdot \mathbf{q}\right)\left(\boldsymbol{\sigma}_{p} \cdot \mathbf{q}\right)}{4 m_{e} m_{p} c^{2} \mathbf{q}^{2}}\right. \\
& \left.\left.+\frac{\boldsymbol{\sigma}_{e} \cdot \boldsymbol{\sigma}_{p}}{4 m_{e} m_{p} c^{2}}\right)+F_{2}^{e} F_{2}^{p}\left(\frac{\boldsymbol{\sigma}_{e} \cdot \boldsymbol{\sigma}_{p}}{4 m_{e} m_{p} c^{2}}-\frac{\left(\boldsymbol{\sigma}_{e} \cdot \mathbf{q}\right)\left(\boldsymbol{\sigma}_{p} \cdot \mathbf{q}\right)}{4 m_{e} m_{p} c^{2} \mathbf{q}^{2}}\right)\right] .
\end{aligned}
$$

If one now takes all form-factors at zero momentum transfer: $F_{1}^{e}(0)=1, F_{2}^{e}(0)=\kappa_{e}=$ 1159.6521811 $(7) \times 10^{-6}, F_{1}^{p}(0)=1, F_{2}^{p}(0)=\kappa_{p}=1.792847351(2)$, the potentials are:

$$
\hat{V}_{\text {fine }}=\frac{e^{2}}{4 m_{e}^{2} c^{2}}\left(\frac{\left(\mathbf{r} \times \hat{\mathbf{p}}_{e}\right)}{r^{3}}\left(1+2 \kappa_{e}\right)-\frac{2 m_{e}}{m_{p}} \frac{\left(\mathbf{r} \times \hat{\mathbf{p}}_{p}\right)}{r^{3}}\left(1+\kappa_{e}\right)\right) . \boldsymbol{\sigma}_{e},
$$

for the fine structure and

$$
\begin{aligned}
& \hat{V}_{\mathrm{hfs}}=\frac{e^{2}}{2 m_{e} m_{p} c^{2}}\left(\frac{\left(\mathbf{r} \times \hat{\mathbf{p}}_{e}\right)}{r^{3}}\left(1+\kappa_{p}\right)-\frac{m_{e}}{2 m_{p}} \frac{\left(\mathbf{r} \times \hat{\mathbf{p}}_{p}\right)}{r^{3}}\left(1+2 \kappa_{p}\right)\right) \cdot \boldsymbol{\sigma}_{p} \\
& +\left(1+\kappa_{e}\right)\left(1+\kappa_{p}\right) \frac{e^{2}}{4 m_{e} m_{p} c^{2}}\left(-\frac{\boldsymbol{\sigma}_{e} \cdot \boldsymbol{\sigma}_{p}}{r^{3}}+3 \frac{\left(\boldsymbol{\sigma}_{e} \cdot \hat{\mathbf{r}}\right)\left(\boldsymbol{\sigma}_{p} \cdot \hat{\mathbf{r}}\right)}{r^{3}}+\frac{8 \pi}{3} \boldsymbol{\sigma}_{e} \cdot \boldsymbol{\sigma}_{p} \delta(\mathbf{r})\right) .
\end{aligned}
$$

for the hyperfine part where we have included some part of the spin-orbit interaction terms. With $e^{2}=\alpha$ and neglecting $\kappa_{e}$ and the term suppressed by $m_{e} m_{p}$, the above fine structure term due to spin orbit coupling (Eq. 22) reduces to

$$
\hat{V}_{\text {fine }}^{\text {book }}=\frac{\alpha}{4 m_{e}^{2} c^{2}}\left(\frac{\left(\mathbf{r} \times \hat{\mathbf{p}}_{e}\right)}{r^{3}}\right) \cdot \boldsymbol{\sigma}_{e}=\frac{\alpha}{2 m_{e}^{2} c^{2}}\left(\frac{\mathbf{L} \cdot \mathbf{S}_{\mathbf{e}}}{r^{3}}\right)
$$

found in books [12] (note that in [12] the notation is slightly different and $e^{2}=4 \pi \epsilon_{0} \alpha$ ). The hyperfine potential after neglecting the $\kappa_{e}$ term, the spin orbit term and the one suppressed by $m_{p}^{2}$, reduces to Eq.(18) which is also a standard result found in books [12]. If we retain the spin orbit term (not suppressed by $m_{p}^{2}$ ) however, we recover

$$
\hat{V}_{\mathrm{hfs}}^{b o o k}=\frac{\alpha}{m_{e} m_{p} c^{2}} \frac{g_{p}}{2}\left[\frac{\left(\mathbf{r} \times \hat{\mathbf{p}}_{e}\right)}{r^{3}} \cdot \mathbf{S}_{\mathbf{p}}+\frac{\mathbf{3}\left(\mathbf{S}_{\mathbf{e}} \cdot \hat{\mathbf{r}}\right)\left(\mathbf{S}_{\mathbf{e}} \cdot \hat{\mathbf{r}}\right)-\mathbf{S}_{\mathbf{e}} \cdot \mathbf{S}_{\mathbf{p}}}{\mathbf{r}^{\mathbf{3}}}+\frac{\mathbf{8} \pi}{\mathbf{3}} \mathbf{S}_{\mathbf{e}} \cdot \mathbf{S}_{\mathbf{p}} \delta^{\mathbf{3}}(\mathbf{r})\right]
$$

which in the absence of any proton structure, i.e., dropping the factor $g_{p} / 2$ is another text book result as in [21]. 
We emphasize again that we have obtained this agreement after retaining the proton form factors at $q^{2}=0$, i.e., $F_{1}^{p}(0)=1$ and $F_{2}^{p}(0)=\kappa_{p}$ which is not a small number. $F_{2}^{p}(0)$ would be tiny if the proton would be a point-like particle. This implies that the hyperfine potential in books, necessarily includes parts of the finite size corrections (in the form of $\left.F_{2}^{p}(0)\right)$. It is now straightforward to generalize the result to take into account the full finite size corrections to the potentials by keeping the full $q^{2}$ dependence, i.e., using $F_{1}^{p}\left(q^{2}\right)$ and $F_{2}^{p}\left(q^{2}\right)$.

\section{HYPERFINE SPLITTING IN $e^{-} p$ AND $\mu^{-} p$ ATOMS}

Having discussed the complete Breit potential with electromagnetic form factors, we shall now focus on the part of the potential which gives rise to the hyperfine splitting in hydrogen atom. We do this with the objective of evaluating the finite size corrections (FSC) to the hyperfine energy levels and comparing them with precision data which is available for the $l=0$ and $l=1$ levels. As outlined in the previous section, normally terms suppressed by small factors arising due to large hadron masses in the denominator are neglected. In the calculations to follow, we shall neglect the term proportional to $1 / m_{p}^{2}$ in the hyperfine

potential. The factor $1 / m_{p}^{2}$ appears in the spin-orbit term which in any case is either small (for $l=1$ ) or not relevant (for $l=0$ ).

\section{A. Hyperfine potential with form factors}

The hyperfine potential in momentum space contains the proton spin-orbit interaction and the proton-electron (or proton-muon in the case of $\mu^{-} p$ ) spin-spin interaction which are responsible for the hyperfine structure.

$$
\begin{array}{r}
\left.\hat{U}(\mathbf{q})_{h f_{s}}=\pi \alpha\left[\frac{\left(\boldsymbol{\sigma}_{X} \cdot \boldsymbol{\sigma}_{p}\right)}{m_{X} m_{p} c^{2}}-\frac{\left(\boldsymbol{\sigma}_{X} \cdot \mathbf{q}\right) \cdot\left(\boldsymbol{\sigma}_{p} \cdot \mathbf{q}\right)}{m_{X} m_{p} c^{2} \mathbf{q}^{2}}\right]\left[\left(F_{1}^{X}+F_{2}^{X}\right)\left(F_{1}^{p}\left(\mathbf{q}^{2}\right)+F_{2}^{p}\left(\mathbf{q}^{2}\right)\right)\right](26){ }^{2}\right) \\
\left.-\left[(2 \pi \alpha) \frac{i \boldsymbol{\sigma}_{p}\left(\mathbf{q} \times \mathbf{p}_{X}\right)}{m_{X} m_{p} c^{2} \mathbf{q}^{2}}\right]\left[F_{1}^{X}\left(F_{1}^{p}\left(\mathbf{q}^{2}\right)+F_{2}^{p}\left(\mathbf{q}^{2}\right)\right)\right]+\left[(2 \pi \alpha) \frac{i \boldsymbol{\sigma}_{p}\left(\mathbf{q} \times \mathbf{p}_{p}\right)}{m_{p}^{2} c^{2} \mathbf{q}^{2}}\right] F_{1}^{X} F_{2}^{p}\left(\mathbf{q}^{2}\right)\right),
\end{array}
$$

with, $X=e, \mu$ for the electron or muon, $F_{1}^{X}=1$ and $F_{2}^{X}=\kappa_{X}$ (the anomalous magnetic moment). $F_{1}^{p}\left(\mathbf{q}^{2}\right)$ and $F_{2}^{p}\left(\mathbf{q}^{2}\right)$ are the two electromagnetic form factors of the proton. Early experiments on electron-proton elastic scattering showed that the cross sections can be written in terms of two form factors, $G_{E}^{p}\left(q^{2}\right)$ and $G_{M}^{p}\left(q^{2}\right)$, where $q^{2}=q_{0}^{2}-\mathbf{q}^{2}$. These Sachs 
form factors [22] can be interpreted in the Breit frame to be the Fourier transforms of the charge $\left(\rho_{C}\right)$ and magnetization $\left(\rho_{M}\right)$ distributions in the proton:

$$
\begin{aligned}
G_{E}^{p}\left(q^{2}\right) & =\int \rho_{C}(\mathbf{r}) e^{-i \mathbf{q} \cdot \mathbf{r}} d \mathbf{r} \\
G_{M}^{p}\left(q^{2}\right) & =\mu_{p} \int \rho_{M}(\mathbf{r}) e^{-i \mathbf{q} \cdot \mathbf{r}} d \mathbf{r} .
\end{aligned}
$$

They can be approximated fairly well by a dipole form [23] as follows:

$$
G_{D}\left(q^{2}\right)=\frac{1}{\left(1+q^{2} / m^{2}\right)^{2}} \approx G_{E}^{p}\left(q^{2}\right) \approx \frac{G_{M}^{p}\left(q^{2}\right)}{\mu_{p}},
$$

where $m^{2}=0.71 \mathrm{GeV}^{2}$. They are related to $F_{1}^{p}\left(q^{2}\right)$ and $F_{2}^{p}\left(q^{2}\right)$ as,

$$
\begin{array}{r}
G_{E}^{p}\left(q^{2}\right)=F_{1}^{p}\left(q^{2}\right)+\frac{q^{2}}{4 m_{p}^{2} c^{2}} F_{2}^{p}\left(q^{2}\right), \\
G_{M}^{p}\left(q^{2}\right)=F_{1}^{p}\left(q^{2}\right)+F_{2}^{p}\left(q^{2}\right) .
\end{array}
$$

Picking up the first terms in the round brackets following $F_{1}^{e} F_{1}^{p}$ and $F_{1}^{e} F_{2}^{p}$ in the Breit potential in (21) and putting them together, one gets (using $q^{2} \approx-\mathbf{q}^{2}$ which is the standard non-relativistic approximation to derive potentials from amplitudes [25]),

$$
-\frac{4 \pi \alpha}{\mathbf{q}^{2}}\left[F_{1}^{p}\left(\mathbf{q}^{2}\right)-\mathbf{q}^{2} \frac{F_{2}^{p}\left(\mathbf{q}^{2}\right)}{4 m_{p}^{2} c^{2}}\right]=-\frac{4 \pi \alpha}{\mathbf{q}^{2}} G_{E}^{p}\left(\mathbf{q}^{2}\right),
$$

which is the standard definition in $\mathbf{q}$-space for the Coulomb potential due to the finite size of the proton. Thus,

$$
\begin{aligned}
F_{1}^{p}\left(\mathbf{q}^{2}\right)= & \frac{1}{\left(1+\mathbf{q}^{2} / m^{2}\right)^{2}}\left[1+\kappa_{p} \frac{\mathbf{q}^{2}}{4 m_{p}^{2}+\mathbf{q}^{2}}\right] \\
& F_{2}^{p}\left(\mathbf{q}^{2}\right)=\frac{1}{\left(1+\mathbf{q}^{2} / m^{2}\right)^{2}} \frac{4 m_{p}^{2} \kappa_{p}}{4 m_{p}^{2}+\mathbf{q}^{2}}
\end{aligned}
$$

where $m_{p}$ is the mass of the proton, $\left(1+\kappa_{p}\right)=\mu_{p}=2.793$ its magnetic moment and $m^{2}=$ $0.71 \mathrm{GeV}^{2}$.

The nuclear finite size corrections (FSC) mentioned in the beginning are thus rooted in the two form factors $F_{1}^{p}$ and $F_{2}^{p}$. The nuclear uncertainty in general can be traced back to (i) different methods used to calculate the FSC (the Breit equation formalism being one such method which, however, is derived directly from quantum field theory) and (ii) the limited precision in the measurement of the two proton form factors. In the present work, we shall restrict ourselves to the dipole form of the form factors given above. The reason for using 
this approximation is that (i) the potentials as well as corrections to the energy levels can be evaluated analytically and (ii) the objective of this work is to present the new method of the Breit equation for calculating the FSC and not explore the uncertainties introduced due to the use of different parametrizations [24] of the form factors.

Replacing (30) in (26) and taking the Fourier transform, the potential for the hyperfine structure in $r$-space is given as,

$$
\hat{V}_{h f s}(r)=\frac{\alpha \mu_{p}}{4 r^{3} m_{X} m_{p} c^{2}}\left[\mu_{X}\left\{3\left(\boldsymbol{\sigma}_{X} \cdot \hat{\mathbf{r}}\right)\left(\boldsymbol{\sigma}_{p} \cdot \hat{\mathbf{r}}\right) f_{1}(r)-\boldsymbol{\sigma}_{X} \cdot \boldsymbol{\sigma}_{p} f_{2}(r)\right\}+2 \mathbf{L} \cdot \boldsymbol{\sigma}_{p} f_{3}(r)\right],
$$

where, $\mu_{X}=1+\kappa_{X}$,

$$
\begin{gathered}
f_{1}(r)=1-e^{-m r}(1+m r)-\frac{m^{2} r^{2}}{6} e^{-m r}(3+m r), \\
f_{2}(r)=f_{1}(r)-\left(m^{3} r^{3} / 3\right) e^{-m r} \text { and } \\
f_{3}(r)=1-e^{-m r}(1+m r)-\frac{m^{2} r^{2}}{2} e^{-m r}
\end{gathered}
$$

For $l=0$ and point like protons, i.e., replacing $F_{1}^{p}=1$ and $F_{2}^{p}=\kappa_{p}$, the Fourier transform of the potential (26) leads to the standard text book potential [25] discussed in the previous section. Thus

$$
V_{h f s}^{\text {point }}(r)=\frac{\alpha}{4 m_{X} m_{p} c^{2}} \mu_{X} \mu_{p}\left[\frac{3\left(\boldsymbol{\sigma}_{X} \cdot \hat{\mathbf{r}}\right)\left(\boldsymbol{\sigma}_{p} \cdot \hat{\mathbf{r}}\right)}{r^{3}}-\frac{\boldsymbol{\sigma}_{X} \cdot \boldsymbol{\sigma}_{p}}{r^{3}}+\frac{8 \pi}{3} \boldsymbol{\sigma}_{X} \cdot \boldsymbol{\sigma}_{p} \delta(\mathbf{r})\right],
$$

is similar to (23) except for the first two terms in (23) corresponding to the spin-orbit coupling which is absent in the $l=0$ case. Since in the ground state $(l=0)$, the wave function is spherically symmetric, the expectation value of the first two terms in (32) vanishes and the third term with the delta function contributes to the energy $E_{h f s}^{\text {point }}$ of the $l=0$ hyperfine levels. However, once we include the form factors of the proton which are smooth functions of $q^{2}$, there is no singularity or a delta function term and both terms in the curly bracket of (31) contribute to $E_{h f s}$ for $l=0$. Eq. (31) contains the standard hyperfine structure plus terms involving the FSC and anomalous magnetic moments of the electron or muon.

\section{B. Finite size corrections to the energy levels}

For any $n$ and $l=0$ for example, in case of the hydrogen atom, we need to evaluate the expectation value, $\left.\left\langle\left[3 \boldsymbol{\sigma}_{e} \cdot \hat{\mathbf{r}}\right)\left(\boldsymbol{\sigma}_{p} \cdot \hat{\mathbf{r}}\right) f_{1}(r)-\left(\boldsymbol{\sigma}_{e} \cdot \boldsymbol{\sigma}_{p}\right) f_{2}(r)\right] / r^{3}\right\rangle$. Using the fact that 
$\int(\mathbf{a} \cdot \hat{\mathbf{r}})(\mathbf{b} \cdot \hat{\mathbf{r}}) \sin \theta d \theta d \phi=(4 \pi / 3)(\mathbf{a} \cdot \mathbf{b})$, the energy of the hyperfine levels as evaluated from (31) for any $n$ and $l=0$ is given as,

$$
\begin{array}{r}
E_{h f s}^{n, l=0}=\frac{\alpha}{4 m_{e} m_{p} c^{2}}\left(1+\kappa_{e}\right) \mu_{p}\left\langle\boldsymbol{\sigma}_{e} \cdot \boldsymbol{\sigma}_{p}\right\rangle \frac{m^{3}}{3}\left(\frac{2}{n a}\right)^{3} \frac{\Gamma(n+1)}{2^{2 n-1} n(n !)} \\
\times \sum_{k=0}^{n-1}\left(\begin{array}{c}
2 n-2 k-2 \\
n-k-1
\end{array}\right) \frac{\Gamma(2 k+3)}{k ! \Gamma(k+2)}\left(\frac{n a}{2+m n a}\right)^{3}{ }_{2} F_{1}\left(-2 k, 3 ; 3 ; \frac{4}{2+m n a}\right),
\end{array}
$$

where, $a=1 /\left(\alpha m_{r}\right)$ is the Bohr radius with the reduced mass $m_{r}$ and ${ }_{2} F_{1}$ is the confluent hypergeometric function of the second kind. Replacing the series

$$
{ }_{2} F_{1}(a, b ; c ; z)=1+(a b / c)(z / 1 !)+[a(a+1) b(b+1)] /[c(c+1)]\left(z^{2} / 2 !\right)+\ldots \ldots
$$

in (33) and evaluating for $n=1$ and $n=2$, the energies of the $(1 S)$ and $(2 S)$ hyperfine levels in hydrogen are given as,

$$
\begin{aligned}
& E_{h f s}^{1 S}=\alpha^{4}\left(\frac{m_{r}^{3}}{4 m_{e} m_{p} c^{2}}\right) \mu_{e} \mu_{p}\left\langle\boldsymbol{\sigma}_{e} \cdot \boldsymbol{\sigma}_{p}\right\rangle \frac{8}{3}\left[1-3\left(\frac{2}{m a}\right)+6\left(\frac{2}{m a}\right)^{2}+\ldots\right] \\
& E_{h f s}^{2 S}=\alpha^{4}\left(\frac{m_{r}^{3}}{m_{e} m_{p} c^{2}}\right) \mu_{e} \mu_{p}\left\langle\boldsymbol{\sigma}_{e} \cdot \boldsymbol{\sigma}_{p}\right\rangle \frac{1}{3}\left[1-6\left(\frac{1}{m a}\right)+21\left(\frac{1}{m a}\right)^{2}+\ldots\right] .
\end{aligned}
$$

These are the energies of the hyperfine levels as obtained from the Breit equation for the e $p \rightarrow$ e $p$ amplitude including the proton form factors $F_{1}^{p}\left(\mathbf{q}^{2}\right), F_{2}^{p}\left(\mathbf{q}^{2}\right)$ ( as in Eq. (30)) and $F_{1}^{e}=1, F_{2}^{e}=\kappa_{e}$ at the two vertices. In the absence of the proton FSC, i.e., for $m \rightarrow \infty$ in (34) and not taking into account the correction due to the anomalous electron magnetic moment (i.e. $\kappa_{e} \rightarrow 0$ ), the energies in Eq. (334) go over to the expressions in standard text books [12, 21]. In the next section we shall present a comparison of the corrections evaluated using the above equations with accurate data available for the $1 S$ and $2 S$ hyperfine levels.

In [19], the size of the proton was deduced from a measurement of the muonic Lamb shift. The total predicted $2 \mathrm{~S}_{1 / 2}^{f=1}-2 \mathrm{P}_{1 / 2}^{f=2}$ energy difference is a sum of contributions from the fine and hyperfine splittings among others. The authors claimed a high accuracy on the radius deduced from this energy difference and concluded that the Rydberg constant has to be shifted by $-110 \mathrm{kHz} / \mathrm{c}$. In view of these results it becomes important to estimate the proton FSC to the hyperfine splitting relevant to this transition in the most sophisticated way possible, within a given approach. Furthermore, one should take into account the uncertainties arising due to the use of different approaches. Hence, we also evaluate corrections for the $2 \mathrm{~S}$ and $2 \mathrm{P}$ hyperfine levels in muonic hydrogen using the Breit potential with form factors. 
The energy $E_{h f s}^{2 P}$ evaluated using the potential in Eq. (31) for the triplet spin states is,

$$
\begin{aligned}
E_{h f s}^{2 P}= & \frac{\alpha \mu_{p}}{24 a^{3} m_{\mu} m_{p} c^{2}}\left[\frac{-\mu_{\mu}}{4 j(j+1)}\left(j(j+1)-l(l+1)-\frac{3}{4}\right)\left(f(f+1)-j(j+1)-\frac{3}{4}\right)\right. \\
& \times\left[1-g_{1}(m a)-2 g_{2}(m a)\right]+g_{2}(m a)+\frac{1}{4 j(j+1)}\left(j(j+1)+l(l+1)-\frac{3}{4}\right) \\
& \left.\times\left(f(f+1)-j(j+1)-\frac{3}{4}\right)\left[1-g_{1}(m a)\right]\right]
\end{aligned}
$$

where,

$$
g_{1}(m a)=\tilde{f}^{2}(m a)+2 m a \tilde{f}^{2}(m a)+3 m^{2} a^{2} \tilde{f}^{4}(m a)
$$

and

$$
g_{2}(m a)=2 m^{3} a^{3} \tilde{f}^{5}(m a) \text { with } \tilde{f}(m a)=1 /(1+m a) .
$$

The quantum numbers $f, j$ and $l$ refer to the total angular momentum of the system, $f=j+s_{p}$, the electron total angular momentum, $j=l+s_{e}$ and the orbital angular momentum $l$. The expressions for any $n$ and $l$ contain the hypergeometric functions ${ }_{2} F_{1}$ as before. Since these expressions are extremely lengthy, we present them in the appendix and here we write the result in the form of Eq.(35) using a truncated expansion of ${ }_{2} F_{1}$ as done before Eqs (34).

\section{RESULTS AND DISCUSSIONS}

The frequencies of the $l=0$ hyperfine intervals in hydrogen atom have been measured quite accurately. The precise value of the $(2 S)$ splitting in literature is 177556834.3(6.7) $\mathrm{kHz}$ [26] and that of the $(1 S)$ is $1420405.751768(1) \mathrm{kHz}$ [27]. In Table I we present the corrections to the frequencies calculated using Eqs (34) for the hyperfine energies. Denoting the hyperfine energy with $\kappa_{e} \rightarrow 0$ and $m \rightarrow \infty$ as $E^{0}$, one with only $m \rightarrow \infty$ as $E^{\text {noFSC }}$, the FSC correction introduced due to the proton form factors is denoted as $\Delta E^{F S C}=$ $E_{h f s}^{n S}-E^{n o F S C}$. The correction due to the proton form factors as well as the anomalous magnetic moment of the electron is denoted as $\Delta E^{\text {corr }}=E_{h f s}^{n S}-E^{0}$.

One can see that the FSC are large and relevant considering the accuracy of the experimental numbers. The FSC are often included via a multiplicative factor containing form factors [18, 28, 29] or more simply by introducing a correction depending on the proton radius [30]. One expects [31] the difference $D_{21}=8 E_{h f s}^{2 S}-E_{h f s}^{1 S}$ to be free of proton finite 
TABLE I: Frequencies in kHz of hyperfine splittings in hydrogen atom.

\begin{tabular}{|c|c|c|c|}
\hline$(\mathrm{nl})$ & $f^{0}$ & $\Delta f^{F S C}$ & $\begin{array}{c}\Delta f^{\text {corr }} \\
\text { due to proton structure }\end{array}$ \\
\hline$(1 \mathrm{~S})$ & 1418840.09 & -37.696 & 1607.665 \\
\hline$(2 \mathrm{~S})$ & 177355.01 & -4.712 & 200.958 \\
\hline
\end{tabular}

size corrections. From Eqs (34) one can see that this difference is finite but small and we find $\Delta D_{21}=-0.0833 \mathrm{~Hz}$.

\section{A. Comparison with other methods}

The need to include structure corrections to the hyperfine energy levels due to the finite size of the proton in the hydrogen atom was noticed about 50 years ago. After the pioneering work of Zemach [18] many other calculations followed. In what follows, we first summarize the main aspects of the Breit potential method and then present a comparison with other methods in literature.

\section{Breit potential method}

This method follows the standard method of quantum field theory to calculate a potential as the Fourier transform of an elastic amplitude. As mentioned in the introduction, it is a well documented method and widely used in different branches of physics. The finite size corrections (FSC) to the potential stem from replacing the point-like vertex in the one photon exchange amplitude (the latter without form factors gives rise to the hyperfine Hamiltonian in the standard Breit equation) by the standard vertex which includes form factors. The result thus generalizes the standard Breit equation to encode all necessary FSC to the potential. It should be emphasized again that the standard Breit equation gives all operators (fine and hyperfine) which appear in the Hamiltonian for the hydrogenic atom. It is therefore true to say that the fine and hyperfine operators come directly from quantum field theory. A perturbation of these operators by the electromagnetic form factors seems then to be the most natural way to include FSC. In the second step we use the unperturbed Coulomb wave functions in the time-independent perturbation theory to arrive at the FSC 
to the energy levels. Thus the correction to the hyperfine energy level, $\Delta E_{h f s}$, is given by, $\Delta E_{h f s}=E_{h f s}-E_{h f s}^{n o F S C}$, where

$$
E_{h f s}=\int \Psi^{*}(\mathbf{r}) V_{h f s}(r) \Psi(\mathbf{r}) d \mathbf{r} .
$$

$\Psi(\mathbf{r})$ is the usual hydrogen atom wave function (solution of the unperturbed Hamiltonian) and $V_{h f s}(r)$ is the hyperfine potential with form factors (as given in Eq.(31)). $E_{h f s}^{n o F S C}$ is evaluated as in (36), however, with the potential $V_{h f s}(r)$ replaced by $V_{h f s}^{\text {point }}(r)$. Note that this method involves a systematic expansion in $\alpha$. This is to say, if an operator being part of the Hamiltonian without FSC is of order $\alpha^{n}$, the corresponding FSC to the potential will also come out to be of the same order.

\section{Zemach's original work}

The approach of Zemach as presented in [18] is very different from that of the Breit potential method described above. The hyperfine Hamiltonian is not derived via the Breit equation and hence the FSC are not obtained as perturbations to the Breit equation. Zemach starts directly deriving the hyperfine energy shift $\Delta E_{Z}$ by introducing a magnetic field $\mathbf{H}(\mathbf{r})$ such that,

$$
\Delta E_{Z}=\mu_{1} \int \Psi_{Z}^{*}(\mathbf{r})\left\langle\boldsymbol{\sigma}_{2} \cdot \mathbf{H}(\mathbf{r})\right\rangle \Psi_{Z}(\mathbf{r})
$$

where part of the FSC has already been incorporated in the wave function $\Psi_{Z}$ which is a solution of the Schödinger equation for an electron moving in the field of the distribution $e f_{e}(\mathbf{r}) . \mu_{1}$ denotes the electron magnetic moment and $f_{e}(\mathbf{r})$ is the Fourier transform of one of the proton's form factors $F_{1}\left(q^{2}\right)$. The magnetic field $\mathbf{H}(\mathbf{r})$ at a given point $\mathbf{r}$ which is usually the field due to a point magnetic dipole is then modified to include the Fourier transform of the magnetic Sachs form factor $G_{M}\left(q^{2}\right)$ mentioned earlier in the article. As a result, Zemach finally obtains,

$$
\Delta E_{Z}=-\frac{2}{3} \mu_{1} \mu_{2}\left\langle\boldsymbol{\sigma}_{1} \cdot \boldsymbol{\sigma}_{2}\right\rangle \int\left|\Psi_{Z}(\mathbf{r})\right|^{2} f_{m}(\mathbf{r}) d \mathbf{r}
$$

where $\mu_{1,2}=e_{1,2} / 2 m_{1,2}$ and $f_{m}(\mathbf{r})$ is the Fourier transform of $G_{M}\left(q^{2}\right)$.

A comparison of Eqs (37) and (38) with Eq. (36) makes it obvious that the Zemach and Breit approaches are completely different. Whereas the Breit approach involves the expectation value (using unperturbed wave functions) of a hyperfine potential with form factors, the Zemach approach has the proton structure information embedded in the wave 
function $\Psi_{Z}(\mathbf{r})$ (in terms of the $F_{1}\left(q^{2}\right)$ form factor) as well as the magnetic field (in the form of $\left.G_{M}\left(q^{2}\right)\right)$.

In the same work, following a perturbative formalism as given by Karplus and Klein, Zemach obtains another expression for $\Delta E_{Z}$ (Eq. (5.8) in [18]) which apart from a small difference in the use of the reduced mass is nothing but the momentum space representation of (38). Performing an explicit comparison of this expression with (36) by rewriting (36) in momentum space using (26) it is easy to see that there arise some additional terms in the Breit method as compared to the Zemach approach.

Zemach further introduced some approximations and a new distribution function $f_{\text {em }}(\mathbf{r})=$ $\int f_{e}(\mathbf{r}-\mathbf{s}) f_{m}(\mathbf{s}) d \mathbf{s}$ to obtain the well known Zemach formula, $\Delta E_{Z}=\Delta E_{0}\left(1-2 m_{1} \alpha\langle r\rangle_{e m}\right)$ where $\langle r\rangle_{e m}=\int r f_{e m}(\mathbf{r}) d \mathbf{r}$.

\section{Bodwin and Yennie's correction}

In [32], in addition to computing the recoil corrections to the hydrogen hyperfine splitting, the authors provided a formalism to obtain the proton structure corrections to the hyperfine splitting in hydrogen. The theoretical procedure involves the evaluation of perturbation kernels corresponding to structure dependent photon-proton vertices. A given perturbation kernel does not yield a result of unique order in $\alpha$ but the kernels are ranked in importance according to the largest contribution that they produce. It was found that it was sufficient to include the contribution of the one loop kernel to the hfs. The six term lengthy expression for $\Delta E_{h f s}$ was analysed and the first term after some approximations was found to reproduce the Zemach formula. Thus the Zemach correction within the formalism of Bodwin and Yennie was given as,

$$
\Delta E=E_{F} \frac{2 \alpha m_{r}}{\pi^{2}} \int d^{3} p \frac{1}{\left(\mathbf{p}^{2}+\gamma^{2}\right)^{2}}\left[\frac{G_{E}\left(-\mathbf{p}^{2}\right) G_{M}\left(-\mathbf{p}^{2}\right)}{1+\kappa}-1\right],
$$

where $E_{F}$ is the Fermi energy and we refer the reader to [32] for details of the notation. The authors found that the net contribution of the remaining five terms in their expression was small as compared to the Zemach correction. A comparison of the results of the present work and corrections obtained using other methods is presented at the end of this section.

A related approach was presented in [28] where the Zemach correction was reproduced in the limit of large proton mass. The order $\alpha^{5}$ corrections to the hyperfine energy levels 
with proton structure included were evaluated from two photon exchange amplitudes. In Zemach's original work both one and two photon exchanges between the electron and proton were considered. Zemach noted that for the two photon exchange case the interaction is of second order in $\alpha$ (i.e. one order higher than the one photon exchange interaction) and that the contribution of this term is very small. The Breit potential used in time-independent perturbation theory gives the $\alpha^{5}$ and higher order corrections to the energy from the one photon exchange diagram (this potential is first order in $\alpha$ as in Zemach's work) . Clearly approaches such as in [28] will differ from the Breit method and lead to different results for the FSC.

\section{Order $\alpha^{5}$ corrections with one and two photon exchange}

Note that the corrections obtained in the present work are the finite size corrections to the hyperfine part of the Breit Hamiltonian. Once the $q^{2}$ dependent form factors are replaced by those at $q^{2}=0$, one recovers the standard hyperfine Hamiltonian for point particles. This is different from some of the methods mentioned above which evaluate the finite size and recoil corrections within the same formalism. They recover the recoil correction to the hyperfine structure on substituting form factors at $q^{2}=0$ in the full expression (which involves the Zemach plus recoil corrections) [28].

We emphasize here that the approach in the present work is conceptually quite different from the ones above which are based on the Bethe Salpeter equation. There one starts by writing the hyperfine energy contribution (at order $\alpha^{5}$ ) induced by a skeleton diagram with two photon exchange. This consists of a divergent integral, namely,

$$
\frac{8 Z \alpha}{\pi n^{3}} E_{F} \int \frac{d k}{k^{2}}
$$

where $E_{F}$ is the Fermi energy and is of order $\alpha^{4}$. Insertion of the electromagnetic form factors leads to the correction (Zemach term of Bodwin and Yennie),

$$
\Delta E=\frac{8 Z \alpha m}{\pi n^{3}} E_{F} \int \frac{d k}{k^{2}}\left[\left(G_{E}\left(-k^{2}\right)-1\right)+\left(\frac{G_{M}\left(-k^{2}\right)}{1+\kappa}-1\right)\right] .
$$

Note that the introduction of finite size effects through the electromagnetic form factors $G_{E}$ and $G_{M}$ has not changed the order of $\alpha$ in the energy (i.e. to say that the order $\alpha^{5}$ is due to two photon exchange and not due to the introduction of finite size effects). In 
the Breit potential approach of the present work, the hyperfine energy calculated using the potential for point-like protons is of order $\alpha^{4}$ (this is the Fermi energy). The introduction of the potential with form factors (which is of the same order in $\alpha$ as the point potential) to evaluate the energy using first order perturbation theory leads to order $\alpha^{5}$ and higher order corrections terms from the same one photon exchange diagram. The higher orders in $\alpha$ arise due to the perturbation theory approach and not due to more photons being exchanged. This is evident from Eqs (34) where the general expression for the hyperfine energy level for any $n$ and $l=0$ is written in a more lucid way by replacing a series expansion of the confluent hypergeometric functions. One can see (using $\left.a=1 /\left(\alpha m_{r}\right)\right)$ that order $\alpha^{5}$ and higher corrections appear in the same expression obtained from a one photon exchange diagram. Setting $m \rightarrow \infty$ in Eqs (34) in order to remove the effect of proton structure returns the order $\alpha^{4}$ Fermi energy.

\section{Friar's finite size correction}

In passing, we finally note that there exist some other approaches such as the one proposed by Friar [29]. The finite size correction within this approach seems to be independent of the parametrization of the form factors. However, the hydrogen atom wave function is not taken to be $\mathbf{r}$ dependent but rather at $\mathbf{r}=0$. Besides, it also appears that though the charge distribution of the proton is taken into account, the magnetization distribution is omitted.

We end this section by mentioning that the correction of $-58.2 \mathrm{kHz}$ in [29] evaluated to the 1S hyperfine level is very close to $-60.2 \mathrm{kHz}$ obtained in [33]. It differs from the correction of the present work but is of the same order of magnitude. Within the Breit potential approach we obtain the FSC of $-37.696 \mathrm{kHz}$ to the $1 \mathrm{~S}$ level splitting in hydrogen. Changing the FSC of $-60.2 \mathrm{kHz}$ to our value $-37.696 \mathrm{kHz}$ would change the total theoretical hfs value as calculated in [33] (Table 19) from $1420399.3 \mathrm{kHz}$ to $1420421.8 \mathrm{kHz}$. This is important considering the exact experimental value $1420405.751768(1) \mathrm{kHz}$ and the fact that radiative corrections

range in the order $10^{-2}-10^{-3} \mathrm{kHz}$ [33] (Tables 14-15). The FSC to $D_{21}=8 E_{h f s}^{2 S}-E_{h f s}^{1 S}$ is also sensitive to the method used. In [31], the correction, $D_{21}($ nucl $)=-0.002 \mathrm{kHz}$ as compared to $-0.0000833 \mathrm{kHz}$ of the present work. Such a small correction makes $D_{21}$ almost free of finite size effects. The experimental value for $D_{21}$ is $48.923(54) \mathrm{kHz}$ [34]. Fourth order QED corrections are $0.018 \mathrm{kHz}$ [34] and comparable to $-0.002 \mathrm{kHz}$, however, bigger than our 
TABLE II: Energies in meV of hyperfine levels and splittings in muonic hydrogen atom.

\begin{tabular}{|c|c|c|c|}
\hline Level & $E^{0}$ & $\Delta E^{F S C}$ & $\Delta E^{\text {corr }}$ \\
& & due to proton structure & due to $\kappa_{\mu}$ and proton structure \\
\hline $2 \mathrm{~S}_{1 / 2}^{f=1}$ & 5.70135 & -0.0280860 & -0.02144 \\
\hline $2 \mathrm{P}_{1 / 2}^{f=1}$ & 1.90045 & -0.0000064 & 0.00110 \\
\hline $2 \mathrm{P}_{1 / 2}^{f=0}$ & -5.70135 & 0.0000345 & -0.00329 \\
\hline $2 \mathrm{P}_{3 / 2}^{f=2}$ & 1.14027 & -0.0000077 & -0.00034 \\
\hline $2 \mathrm{P}_{3 / 2}^{f=1}$ & -1.90045 & 0.0000103 & 0.00056 \\
\hline $\mathrm{Splitting}$ & & & -0.085753 \\
\hline $2 \mathrm{~S}$ & 22.80541 & -0.112342 & 0.004390 \\
\hline $2 \mathrm{P}_{1 / 2}$ & 7.60180 & -0.000041 & -0.000904 \\
\hline $2 \mathrm{P}_{3 / 2}$ & 3.04072 & -0.000018 & \\
\hline
\end{tabular}

estimate of FSC. From Eqs (34) it is obvious that the FSC to $D_{21}$ begin at order $\alpha^{6}$ and hence are expected to be very small.

\section{B. Hyperfine splitting in muonic hydrogen}

In Table II we present the corrections to the energies calculated using Eq. (34) but for the case of muonic hydrogen $\left(\mu^{-} p\right) 2 \mathrm{~S}$ splitting and Eq. (35) for the $2 \mathrm{P} j(j=1 / 2,3 / 2)$ splitting in $\mu^{-} p$. The individual level energies are also listed. The splitting $2 \mathrm{~S}$ is the difference $2 \mathrm{~S}_{1 / 2}^{f=1}-2 \mathrm{~S}_{1 / 2}^{f=0}$ and the splittings $2 \mathrm{P}_{1 / 2}$ and $2 \mathrm{P}_{3 / 2}$ are $2 \mathrm{P}_{1 / 2}^{f=1}-2 \mathrm{P}_{1 / 2}^{f=0}$ and $2 \mathrm{P}_{3 / 2}^{f=2}-2 \mathrm{P}_{3 / 2}^{f=1}$ respectively. In fact, the energy of the $2 \mathrm{~S}$ splitting is $4 \mathrm{E}_{h f s}\left(2 \mathrm{~S}_{1 / 2}^{f=1}\right)$. In the evaluation of the proton radius in [19], the values of the hyperfine splittings were taken from [35], where the FSC for the $2 \mathrm{~S}$ were evaluated using the Zemach method and those for the $2 \mathrm{P}$ case were not taken into account. Their FSC (taken from Table II of the first reference in [35]) of order $\alpha^{5}$ and $\alpha^{6}$ sum to $-0.1535 \mathrm{meV}$ in contrast to the $-0.11234 \mathrm{meV}$ of the present work. This result would change the input of $\Delta E_{H E S}^{2 S}=22.8148 \mathrm{meV}$ (which includes the FSC corrections using Zemach's method) used in [19] to $\Delta E_{H F S}^{2 S}=22.8560 \mathrm{meV}$. Correcting the $2 \mathrm{P}$ hyperfine splitting in [19], $\Delta E_{H F S}^{2 P_{3 / 2}}=3.392588 \mathrm{meV}$ would change to $\Delta E_{H F S}^{2 P_{3 / 2}}=3.392570$ $\mathrm{meV}$. These corrections are quite relevant considering the precision taken into account in 
[19] while deducing the charge radius of the proton.

\section{SUMMARY}

To summarize, the Breit potential for hydrogen atom with the inclusion of electromagnetic form factors of the proton is presented. This includes the proton structure corrections to the standard Coulomb potential plus terms such as the Darwin term (which takes into account the relativistic effects), the spin-spin and spin-orbit interaction terms (corresponding to fine and hyperfine structure) and retarded potential terms. We focused in particular on certain terms involving the spin-spin and spin-orbit interaction with the aim of studying the proton form factor effects (or proton finite size effects) in the hyperfine splitting in electronic and muonic hydrogen. The finite size corrections (FSC) to the potentials originate from the way the photon interacts with extended objects. It is therefore possible to include such corrections already in the standard Breit equation where the potential is of order $\alpha$. It is important to note that the standard hyperfine Hamiltonian for hydrogen atom follows naturally from the expressions for the Breit potential with form factors, once the form factors are replaced by their values at $q^{2}=0$. This Hamiltonian contains $\kappa_{p}\left(=F_{2}^{p}(0)\right)$, the anomalous magnetic moment of the proton which is large due to the fact that the proton is an extended object. Hence part of the finite size correction is already inherent in the hyperfine Hamiltonian. By including $q^{2}$ dependent form factors, we just make the Hamiltonian more general and complete. We presented here the FSC for three cases: 1S and $2 \mathrm{~S}$ splittings in hydrogen atom, Sternheim's observable $D_{21}$ and $2 \mathrm{~S}$ and $2 \mathrm{P}$ splittings in muonic hydrogen. The theoretical aspects as well as numerical values obtained in the present work were compared with other existing methods for FSC in literature. The present work aims also at showing that there exist different sophisticated and consistent approaches to evaluate the finite size corrections.

Our FSC of $-0.11234 \mathrm{meV}$ to the $2 \mathrm{~S}$ hyperfine splitting in muonic hydrogen is close to the order $\alpha^{5}$ corrections of $-0.183 \mathrm{meV}$ [28] and $-0.1518 \mathrm{meV}$ [35] using other approaches. As one can see, though the three numbers are of the same order of magnitude, they are not equal to each other. Thus, one can say that there is a small uncertainty in the calculation of the finite size effects, introduced due to the differences in the approaches used for FSC.

The present work gives the analytical expressions for the hyperfine potential and energy 
levels including the finite size corrections due to the structure of the proton. This has been achieved using the dipole form of the proton form factors. Note that the inclusion of proton structure effects gives rise to two very different length scales in the same calculations: the atomic one involving the Bohr radius $a$ (which is of the order of $10^{4} \mathrm{fm}$ ) and the nuclear one with $r_{p} \propto 1 / m$ (of the order of $1 \mathrm{fm}$ ). This would make a numerical evaluation of the integrals involved in the calculations tedious and hence an analytical approach as in the present work is preferable.

\section{APPENDIX: Hyperfine energy levels with proton structure corrections for any $n$}

and $l \neq 0$

The hyperfine energy for any $n$ and $l \neq 0$ is evaluated by taking the expectation value of the potential in Eq. (31). We start by rewriting the potential as follows for convenience:

$$
\begin{aligned}
& \hat{V}_{h f s}=\frac{\alpha}{m_{e} m_{p} c^{2}}\left(1+\kappa_{e}\right)\left(1+\kappa_{p}\right)\left[3\left(\mathbf{S}_{e} \cdot \hat{\mathbf{r}}\right)\left(\mathbf{S}_{p} \cdot \hat{\mathbf{r}}\right)\left(\frac{1}{r^{3}}+\frac{h_{1}}{r^{3}}\right)-\left(\mathbf{S}_{e} \cdot \mathbf{S}_{p}\right)\left(\frac{1}{r^{3}}+\frac{h_{2}}{r^{3}}\right)\right] \\
& +\frac{e^{2}}{m_{e} m_{p} c^{2}}\left(\mathbf{L} \cdot \mathbf{S}_{p}\right)\left[\left(1+\kappa_{p}\right)\left(\frac{1}{r^{3}}+\frac{h_{3}}{r^{3}}\right)+\frac{m_{e}}{2 m_{p}} \frac{h_{4}}{r^{3}}\right]
\end{aligned}
$$

where,

$$
\begin{aligned}
h_{1}= & -e^{-m r}(1+m r)-\frac{m^{2} r^{2}}{2} e^{-m r}-\frac{m^{3} r^{3}}{6} e^{-m r} \\
h_{2}= & -e^{-m r}(1+m r)-\frac{m^{2} r^{2}}{2} e^{-m r}-\frac{m^{3} r^{3}}{2} e^{-m r} \\
h_{3}= & -e^{-m r}(1+m r)-\frac{m^{2} r^{2}}{2} e^{-m r} \\
h_{4}= & \left(1+2 \kappa_{p}\right)\left(1+h_{3}\right)+\frac{\kappa_{p}}{\left(1-k^{2}\right)^{2}} e^{-m r}(1+m r)+\frac{\kappa_{p}}{1-k^{2}} \frac{m^{2} r^{2}}{2} e^{-m r} \\
& -\frac{\kappa_{p}}{\left(1-k^{2}\right)^{2}} e^{-m k r}(1+m k r),
\end{aligned}
$$

and $k=2 m_{p} / m$. Eq. (A-1) was obtained using the identity,

$$
\left(\hat{\boldsymbol{\sigma}}_{e} \cdot \vec{\nabla}\right)\left(\hat{\boldsymbol{\sigma}}_{p} \cdot \vec{\nabla}\right) G(r)=\left(\hat{\boldsymbol{\sigma}}_{e} \cdot \hat{\boldsymbol{\sigma}}_{p}\right)\left(\frac{1}{r} \frac{\partial G(r)}{\partial r}\right)+\frac{\left(\hat{\boldsymbol{\sigma}}_{e} \cdot \mathbf{r}\right)\left(\hat{\boldsymbol{\sigma}}_{p} \cdot \mathbf{r}\right)}{r^{2}}\left(\frac{\partial^{2} G(r)}{\partial r^{2}}-\frac{1}{r} \frac{\partial G(r)}{\partial r}\right) .
$$

Using first order time independent perturbation theory,

$$
E_{h f s}^{n l}=\int \Psi_{n l m}^{*}(\mathbf{r}) \hat{V}_{h f s} \Psi_{n l m}(\mathbf{r}) d \mathbf{r}
$$


where,

$$
\Psi_{n l m}(r, \theta, \phi)=\left[\left(\frac{2}{n a}\right)^{3} \frac{(n-l-1) !}{2 n(n+l) !}\right]^{1 / 2} e^{-r / n a}\left(\frac{2 r}{n a}\right)^{l} L_{n-l-1}^{2 l+1}(2 r / n a) Y_{l}^{m}(\theta, \phi)
$$

is the wave function of the hydrogen atom. The identity,

$$
\left[L_{n-l-1}^{2 l+1}\left(\frac{2 r}{n a}\right)\right]^{2}=\frac{\Gamma(n+l+1)}{2^{2(n-l-1)}(n-l-1) !} \sum_{j=0}^{n-l-1}\left(\begin{array}{c}
2(n-l-j-1) \\
n-l-j-1
\end{array}\right) \frac{(2 j) !}{j ! \Gamma(2 l+j+2)} L_{2 j}^{2(2 l+1)}\left(\frac{4 r}{n a}\right),
$$

allows one to write the expectation value of a function sandwiched between the hydrogen wave functions as,

$$
\begin{aligned}
\langle A\rangle= & \left(\frac{2}{n a}\right)^{2 l+3} \frac{1}{2 n 2^{2(n-l-1)}} \sum_{j=0}^{n-l-1}\left(\begin{array}{c}
2(n-l-j-1) \\
n-l-j-1
\end{array}\right) \frac{(2 j) !}{j ! \Gamma(2 l+j+2)} \\
& \times \int_{0}^{\infty} d r A(r) e^{-2 r / n a} r^{2 l+2} L_{2 j}^{2(2 l+1)}(4 r / n a) .
\end{aligned}
$$

This leads to,

$$
\begin{aligned}
& E_{h f s}^{n l}\left(j, f,\left\langle\mathbf{S}_{e} \cdot \mathbf{S}_{p}\right\rangle\right)=\frac{e^{2}}{m_{e} m_{p}}\left[\left(-\left(1+\kappa_{e}\right)\left(1+\kappa_{p}\right)\left(j(j+1)-l(l+1)-\frac{3}{4}\right)\right.\right. \\
& \left.\times\left\langle\frac{1}{r^{3}}+\frac{h_{1}}{r^{3}}\right\rangle+\left(j(j+1)+l(l+1)-\frac{3}{4}\right)\left\langle\left(1+\kappa_{p}\right)\left(\frac{1}{r^{3}}+\frac{h_{3}}{r^{3}}\right)+\frac{m_{e}}{2 m_{p}} \frac{h_{4}}{r^{3}}\right\rangle\right) \\
& \left.\times\left(\frac{f(f+1)-j(j+1)-\frac{3}{4}}{4 j(j+1)}\right)+\frac{m^{3}}{3}\left\langle\mathbf{S}_{e} \cdot \mathbf{S}_{p}\right\rangle\left\langle e^{-m r}\right\rangle\right],
\end{aligned}
$$

where, the expectation values are:

$$
\begin{aligned}
& \left\langle\frac{1}{r^{3}}+\frac{h_{1}}{r^{3}}\right\rangle=\left(\frac{2}{n a}\right)^{2 l+3} \frac{1}{2 n 2^{2(n-l-1)} \Gamma(4 l+3)} \sum_{j=0}^{n-l-1}\left(\begin{array}{c}
2(n-l-j-1) \\
n-l-j-1
\end{array}\right) \frac{\Gamma(4 l+2 j+3)}{j ! \Gamma(2 l+j+2)} \\
& {\left[\Gamma(2 l)\left(\frac{n a}{2}\right)^{2 l}{ }_{2} F_{1}(-2 j, 2 l ; 4 l+3 ; 2)-\Gamma(2 l)\left(\frac{n a}{2+m n a}\right)^{2 l}{ }_{2} F_{1}\left(-2 j, 2 l ; 4 l+3 ; \frac{4}{2+m n a}\right)\right.} \\
& -m \Gamma(2 l+1)\left(\frac{n a}{2+m n a}\right)^{2 l+1}{ }_{2} F_{1}\left(-2 j, 2 l+1 ; 4 l+3 ; \frac{4}{2+m n a}\right)-\frac{m^{2}}{2} \Gamma(2 l+2) \\
& \left(\frac{n a}{2+m n a}\right)^{2 l+2}{ }_{2} F_{1}\left(-2 j, 2 l+2 ; 4 l+3 ; \frac{4}{2+m n a}\right)-\frac{m^{3}}{6} \Gamma(2 l+3)\left(\frac{n a}{2+m n a}\right)^{2 l+3} \\
& \left.{ }_{2} F_{1}\left(-2 j, 2 l+3 ; 4 l+3 ; \frac{4}{2+m n a}\right)\right], \\
& \left\langle\left(1+\kappa_{p}\right)\left(\frac{1}{r^{3}}+\frac{h_{3}}{r^{3}}\right)+\frac{m_{e}}{2 m_{p}} \frac{h_{4}}{r^{3}}\right\rangle=\left(\frac{2}{n a}\right)^{2 l+3} \frac{1}{2 n 2^{2(n-l-1)} \Gamma(4 l+3)} \\
& \sum_{j=0}^{n-l-1}\left(\begin{array}{c}
2(n-l-j-1) \\
n-l-j-1
\end{array}\right) \frac{\Gamma(4 l+2 j+3)}{j ! \Gamma(2 l+j+2)}\left[\left(1+\kappa_{p}+\frac{m_{e}}{2 m_{p}}\left(1+2 \kappa_{p}\right)\right) \Gamma(2 l)\left(\frac{n a}{2}\right)^{2 l}\right.
\end{aligned}
$$




$$
\begin{aligned}
& { }_{2} F_{1}(-2 j, 2 l ; 4 l+3 ; 2)-\left(1+\kappa_{p}+\frac{m_{e}}{2 m_{p}}\left(1+2 \kappa_{p}-\frac{\kappa_{p}}{\left(1-k^{2}\right)^{2}}\right)\right) \Gamma(2 l)\left(\frac{n a}{2+m n a}\right)^{2 l} \\
& { }_{2} F_{1}\left(-2 j, 2 l ; 4 l+3 ; \frac{4}{2+m n a}\right)-\left(1+\kappa_{p}+\frac{m_{e}}{2 m_{p}}\left(1+2 \kappa_{p}-\frac{\kappa_{p}}{\left(1-k^{2}\right)^{2}}\right)\right) m \Gamma(2 l+1) \\
& \left(\frac{n a}{2+m n a}\right)^{2 l+1}{ }_{2} F_{1}\left(-2 j, 2 l+1 ; 4 l+3 ; \frac{4}{2+m n a}\right)-\left(1+\kappa_{p}+\frac{m_{e}}{2 m_{p}}\left(1+2 \kappa_{p}-\frac{\kappa_{p}}{1-k^{2}}\right)\right) \\
& \frac{m^{2}}{2} \Gamma(2 l+2)\left(\frac{n a}{2+m n a}\right)^{2 l+2}{ }_{2} F_{1}\left(-2 j, 2 l+2 ; 4 l+3 ; \frac{4}{2+m n a}\right)-\frac{m_{e}}{2 m_{p}} \frac{\kappa_{p}}{\left(1-k^{2}\right)^{2}} \Gamma(2 l) \\
& \left(\frac{n a}{2+m k n a}\right)^{2 l}{ }_{2} F_{1}\left(-2 j, 2 l ; 4 l+3 ; \frac{4}{2+m k n a}\right)-\frac{m_{e}}{2 m_{p}} \frac{\kappa_{p} m k}{\left(1-k^{2}\right)^{2}} \Gamma(2 l+1)\left(\frac{n a}{2+m k n a}\right)^{2 l+1} \\
& \left.{ }_{2} F_{1}\left(-2 j, 2 l+1 ; 4 l+3 ; \frac{4}{2+m k n a}\right)\right],
\end{aligned}
$$

and

$$
\begin{aligned}
\left\langle e^{-m r}\right\rangle= & \left(\frac{2}{n a}\right)^{2 l+3} \frac{1}{2 n 2^{2(n-l-1)}} \sum_{j=0}^{n-l-1}\left(\begin{array}{c}
2(n-l-j-1) \\
n-l-j-1
\end{array}\right) \frac{\Gamma(4 l+2 j+3)}{j ! \Gamma(2 l+j+2)} \\
& \frac{\Gamma(2 l+3)}{\Gamma(4 l+3)}\left(\frac{n a}{2+m n a}\right)^{2 l+3}{ }_{2} F_{1}\left(-2 j, 2 l+3 ; 4 l+3 ; \frac{4}{2+m n a}\right) .
\end{aligned}
$$

[1] G. Breit, Phys. Rev. 34, 553 (1929); ibid 36, 383 (1930); ibid 39, 616 (1932); J. R. Oppenheimer, Phys. Rev. 35, 461 (1930).

[2] M. De Sanctis, Eur. Phys. J. A 41, 169 (2009); D. A. Kulikov, R. S. Tutik, Mod. Phys. Lett. A 25, 447 (2010); ibid 23, 1829 (2008).

[3] H. B. G. Casimir and P. Polder, Phys. Rev. 73 (1948) 360; E.M. Lifschitz, JETP Lett.2 (1956) 73; F. Ferrer and J. A. Grifols, Phys. Lett. B460, 371, 1999.

[4] E. A. Uehling, Phys. Rev. 48, 55 (1935); L. Wayne Fullerton and G. A. Rinker, Jr., Phys. Rev. A 13, 1283 (1976).

[5] G. Feinberg and J. Sucher, Phys. Rev. A 166, 1638 (1968); S. D. H. Hsu and P. Sikivie, Phys. Rev. D 49, 4951 (1994).

[6] R. Machleidt, Adv. Nucl. Phys. 19, 181 (1989); J. D. Walecka, Theoretical Nuclear and Subnuclear physics, Second Edition, World Scientific 2004.

[7] J. F Donoghue, Phys. Rev. Lett. 72, 2996, 1994.

[8] V. M. Mostepanenko and I. Yu. Sokolov, Sov. J. Nucl. Phys. 46, 685 (1987); J. A. Grifols and S. Tortosa, Phys. lett. B 328, 98 (1994); F. Ferrer and J. A. Grifols, Phys. Rev. D 58, 096006 
(1998); F. Ferrer and M. Nowakowski, Phys. Rev. D 59, 075009 (1999).

[9] J. A. Grifols, Phys. Lett. B 264, 149 (1991).

[10] F. Ferrer, J. A. Grifols and M. Nowakowski, Phys. Lett. B 446, 111 (1999).

[11] F. Ferrer, J. A. Grifols and M. Nowakowski, Phys. Rev. D 61, 057304 (2000).

[12] D. Griffiths, Introduction to Quantum Mechanics, (Pearson Prentice Hall, NJ, 2005).

[13] M. Nowakowski, N. G. Kelkar and T. Mart, Phys. Rev. C 74, 024323 (2006); N. G. Kelkar and M. Nowakowski, Phys. Lett. B 651, 363 (2007).

[14] G. M. Shore, Nucl. Phys. b717, 86 (2005); R. W. Dunford and R. J. Holt, J. Phys. G: Nucl. Part. Phys. 34, 2099 (2007); M.-A. Bouchiat and C. Bouchiat, Rep. Prog. Phys. 60, 1357 (1997).

[15] P. J. Mohr, B. N. Taylor and D. B. Newell, Rev. Mod. Phys. 80, 633 (2008).

[16] N. F. Ramsey, Quantum Electrodynamics, edited by T. Kinoshita (World Scientific, Singapore), Ch. 13, pp. 673 (1990).

[17] M. M. Sternheim, Phys. Rev. B 138, 430 (1965).

[18] A. C. Zemach, Phys. Rev. 104, 1771 (1956).

[19] R. Pohl, Nature 466, 213 (2010).

[20] C. G. Darwin, Proc. R. Soc. London, Ser. A 118, 654 (1928).

[21] H. A. Bethe and E. E. Salpeter, Quantum Mechanics of One- and Two-Electron Atoms, (Dover, NY, 2008).

[22] C. F. Perdrisat, V. Punjabi and M. Vanderhaeghen, Prog. Part. Nucl. Phys. 59, 694 (2007).

[23] P. E. Bosted et al., Phys. Rev. Lett. 68, 3841 (1992).

[24] W. M. Alberico, S. M. Bilenky, C. Giunti and K. M. Graczyk, Phys. Rev. C 79, 065204 (2009).

[25] V. B. Berestetskii, E. M. Lifshitz and L. P. Pitaevskii, Quantum Electrodynamics, LandauLifshitz Course on Theoretical Physics Vol.4, 2nd edition, Oxford: Butterworth-Heinemann (2007).

[26] N. Kolachevsky et al., Phys. Rev. Lett. 102, 213002 (2009); N. Kolachevsky, M. Fischer, S. G. Karshenboim and T. W. Hänsch, Phys. Rev. Lett. 92, 033003 (2004).

[27] S. G. Karshenboim, Phys. Rep. 422, 1 (2005).

[28] K. Pachucki, Phys. Rev. A 53, 2092 (1996); ibid, 60, 3593 (1999).

[29] J. L. Friar, Ann. Phys. (N. Y.) 122, 151 (1979); J. L. Friar and G. L. Payne, Phys. Lett. B 618, 68 (2005). 
[30] C. Itzykson and J.-B. Zuber, Quantum Field Theory, (Dover, NY, 1980).

[31] S. G. Karshenboim and V. G. Ivanov, Phys. Lett. B 524, 259 (2002).

[32] G. T. Bodwin and D. R. Yennie, Phys. Rev. D 37, 498 (1988).

[33] M. I. Eides, H. Grotch and V. A. Shelyuto, Phys. Rep. 342, 63 (2001).

[34] S. G. Karshenboim, preprint arXiv:1005.4875 (hep-ph).

[35] A. P. Martynenko, Phys. Rev. A 71, 022506 (2005); A. P. Martynenko, Physics of Atomic Nuclei 71, 125 (2008). 